\title{
Comorbidity in patients with asymptomatic AS
}

Prashant Vaishnava, Valentin Fuster, Martin Goldman and Robert O. Bonow

In response to our Perspectives article (Vaishnava, P., Fuster, V., Goldman, M. \& Bonow, R. O. Surgery for asymptomatic degenerative aortic and mitral valve disease. Nat. Rev. Cardiol. 8, 173-177 [2011]) $)^{1}$ on asymptomatic valve disease, Gilmanov et al. (Gilmanov, D., Mazzone, A., Berti, S. \& Glauber, M. Is comorbidity equivalent to symptoms in asymptomatic AS? Nat. Rev. Cardiol. doi:10.1038/nrcardio.2011.203-c1) ${ }^{2}$ correctly note the often insidious and not easily recognized onset of symptoms in aortic stenosis (AS). We agree that subtle and nonspecific symptoms might not be fully acknowledged by patients, or become falsely attributed to the effects of aging, deconditioning, or comorbidities. Gilmanov et al. also suggest that the "extent of comorbidity" could be considered a "symptom equivalent for prognostic stratification." Although we agree that comorbidities are common in patients with severe AS, we would caution against this approach of ascribing symptom equivalency to a heterogeneous group of coexisting illnesses. Rather, we would advocate exercise testing with or without echocardiography to risk-stratify asymptomatic patients with AS, by unmasking symptoms and abnormal hemodynamic responses. ${ }^{3-5}$

The occurrence of exercise-limiting symptoms, such as breathlessness, fatigue at low workload, angina, dizziness, or syncope, predicts the development of symptoms, sudden cardiac death, and the need for aortic valve replacement (AVR) among asymptomatic patients with severe AS. ${ }^{6}$ Like Gilmanov and colleagues, we recognize the potential value of elevated levels of B-type natriuretic peptide in determining the optimum timing of AVR, but caution against firm recommendations for this approach at present. ${ }^{7}$

We agree that minimally invasive openheart operative strategies might be an appropriate strategy in appropriately selected patients requiring surgical AVR. However, we feel strongly that transcatheter AVR is not yet ready for asymptomatic patients until more definitive data on feasibility, safety, and long-term efficacy are established.

Zena and Michael A. Wiener Cardiovascular Institute, and the Marie-Jose and Henry R. Kravis Cardiovascular Health Center, The Mount Sinai School of Medicine, 1 Gustave L. Levy Place, New York, NY 10029, USA

(P. Vaishnava, V. Fuster, M. Goldman). Center for Cardiovascular Innovation, Northwestern University Feinberg School of Medicine, 645 North Michigan Avenue, Chicago, IL 60611 , USA (R. O. Bonow).

Correspondence to:

P. Vaishnava

prashant.vaishnava@mountsinai.org

doi:10.1038/nrcardio.2011.203-c2

Competing interests

The authors declare no competing interests.
Contributions

P. Vaishnava and R. O. Bonow wrote the article. All authors reviewed the article prior to submission.

1. Vaishnava, P., Fuster, V., Goldman, M. \& Bonow, R. O. Surgery for asymptomatic degenerative aortic and mitral valve disease. Nat. Rev. Cardiol. 8, 173-177 (2011).

2. Gilmanov, D., Mazzone, A., Berti, S. \& Glauber, M. Is comorbidity equivalent to symptoms in asymptomatic AS? Nat. Rev. Cardiol. http://dx.doi.org/10.1038/ nrcardio.2011.203-c1.

3. Bonow, R. O. et al. ACC/AHA 2006 guidelines for the management of patients with valvular heart disease: a report of the American College of Cardiology/American Heart Association Task Force on Practice Guidelines (writing committee to revise the 1998 Guidelines for the Management of Patients With Valvular Heart Disease): developed in collaboration with the Society of Cardiovascular Anesthesiologists: endorsed by the Society for Cardiovascular Angiography and Interventions and the Society of Thoracic Surgeons. Circulation 114, e84-e231 (2006).

4. Vahanian, A. et al. Guidelines on the management of valvular heart disease: the Task Force on the Management of Valvular Heart Disease of the European Society of Cardiology. Eur. Heart J. 28, 230-268 (2007).

5. Picano, E., Pibarot, P., Lancellotti, P., Monin, J. L. \& Bonow, R. O. The emerging role of exercise testing and stress echocardiography in valvular heart disease. J. Am. Coll. Cardiol. 54, 2251-2260 (2009).

6. Das, P., Rimington, H. \& Chambers, J. Exercise testing to stratify risk in aortic stenosis. Eur. Heart J. 26, 1309-1313 (2005).

7. Ambler, G. et al. Generic, simple risk stratification model for heart valve surgery. Circulation 112, 224-231 (2005). 